\title{
ON THE WEAK TYPE $(1,1)$ INEQUALITY FOR CONJUGATE FUNCTIONS
}

\author{
BURGESS DAVIS ${ }^{1}$
}

\begin{abstract}
A theorem of Kolmogorov states that there is a positive constant $K$ such that if $\tilde{f}$ is the conjugate function of an integrable real valued function $f$ on the unit circle then $m\{|\tilde{f}| \geqq \lambda\} \leqq$ $K\|f\|_{1} / \lambda, \lambda>0$. It is shown that the smallest possible value for $K$ in this theorem, the so called weak type $(1,1)$ norm of the conjugate function operator, is $\left(1+3^{-2}+5^{-2}+\cdots\right) /\left(1-3^{-2}+5^{-2}-\cdots\right) \approx$ 1.347. This number is also shown to be the weak type $(1,1)$ norm of the Hilbert transform operator on functions defined on the real line. The proof uses P. Lévy's result that Brownian motion in the plane is conformally invariant.
\end{abstract}

1. Introduction. Let $D$ be the open unit disc in the complex plane $C$, $A$ be the unit circle, $S$ be the strip $\{z \in C:-1<\operatorname{Im} z<1\}, R$ be the real numbers, and $U$ be the upper half plane $\{z \in C: \operatorname{Im} z>0\}$.

Lebesgue measure on $R$ will be denoted by $\mu$ and $m$ will stand for linear Lebesgue measure on $A$ divided by $2 \pi$. If $f$ is a function on $R$ we write $\|f\|_{1}=\int_{R}|f| d \mu$, and the Hilbert transform $f$ of integrable functions $f$ is defined by

$$
\tilde{f}(t)=\frac{1}{\pi} P V \int_{-\infty}^{\infty} \frac{f(t-s)}{s} d \mu(s) .
$$

If $f$ is a function on $A$ we write $\|f\|_{1}=\int_{A}|f| d m$, and the conjugate function $\tilde{f}$ of integrable functions $f$ is defined by

$$
\tilde{f}\left(e^{i \theta}\right)=P V \int_{-\pi}^{\pi} f\left(e^{i(\theta-\varphi)}\right) \cot (\varphi / 2) d m(\varphi) .
$$

$\Theta$ will stand for $\left(8 / \pi^{2}\right) \int_{0}^{\infty} a e^{a} d a /\left(1+e^{2 a}\right)=\left(8 / \pi^{2}\right)\left(1-3^{-2}+5^{-2}-\cdots\right)=$ $\left(1-3^{-2}+5^{-2}-\cdots\right) /\left(1+3^{-2}+5^{-2}+\cdots\right)$.

The following theorems will be proved.

Received by the editors August 20, 1973.

AMS (MOS) subject classifications (1970). Primary 42A40; Secondary 42A36, $60 \mathrm{~J} 65$.

Key words and phrases. Conjugate function, weak-type inequality, Brownian motion.

${ }^{1}$ Supported by NSF Grant GP 19222.

(c) American Mathematical Society 1974 
THEOREM 1. If $\tilde{f}$ is the conjugate function of an integrable function $f$ on A then

$$
m\{|\tilde{f}| \geqq \lambda\} \leqq \Theta^{-1}\|f\|_{1} / \lambda, \quad \lambda>0,
$$

and (1) does not hold in general if $\Theta^{-1}$ is replaced by a smaller number.

THEOREM 2. If $\tilde{f}$ is the Hilbert transform of an integrable function $f$ on $R$ then

$$
m\{|\tilde{f}| \geqq \lambda\} \leqq \Theta^{-1}\|f\|_{1} / \lambda, \quad \lambda>0,
$$

and (2) does not hold in general if $\Theta^{-1}$ is replaced by a smaller number.

The existence of a positive constant $K$ such that (1) and (2) hold with $\Theta^{-1}$ replaced by $K$ is due to Kolmogorov. (See [5, p. 134].)

The smallest possible constant $K$ for which $m\left\{|\tilde{f}| \geqq \lambda_{2}\right\} \leqq K\|f\|_{1} / \lambda$ is true for nonnegative functions $f$ is known to be 1 , for both conjugate functions and Hilbert transforms (see [1]). Pichorides [3] finds best constants in some strong type conjugate function and Hilbert transform inequalities.

An easy example shows that $\Theta^{-1}$ cannot be replaced by a smaller number in (1). Let $G(z)=(2 / \pi) \log [(i z-1) /(z-i)]-i$. Then $G(0)=0$, $G$ maps $D$ onto $S$ and maps $A$ onto the boundary of $S$. Let $g\left(e^{i \theta}\right)=$ $\operatorname{Re} G\left(e^{i \theta}\right)$. It can be checked that $\|g\|_{1}=\Theta$, and $\tilde{g}\left(e^{i \theta}\right)=\operatorname{Im} G\left(e^{i \theta}\right)$ so that $m\{z \in A:|\tilde{g}(z)|=1\}$. Thus, if $\lambda=1$ and $f=g$, (1) is an equality.

I want to thank D. L. Burkholder for a number of helpful suggestions.

2. Preliminary lemmas. In this section $Z_{t}=X_{t}+i Y_{t}, 0 \leqq t<\infty$, will be a continuous two dimensional Brownian motion. If $z \in \bar{C}, P_{z}$ and $E_{z}$ will stand for probability and expectation associated with $Z_{t}$ given $P\left(Z_{0}=z\right)=1$. $\tau$ will be the first time $\left|Y_{t}\right|=1$. By the result of Kakutani which equates hitting probabilities of Brownian motions and harmonic measures (see [2]), the distribution of $Z_{\tau}$ under $P_{0}$ is harmonic measure on the boundary of $S$ relative to 0 . Thus the distribution of $X_{\tau}$ is given by the density $e^{\pi t / 2} /\left(1+e^{\pi t}\right),-\infty<t<\infty$, so that

$$
E_{0}\left|X_{\tau}\right|=\Theta .
$$

LEMMA 1. If $r$ and $s$ are real numbers and $-1 \leqq s \leqq 1$ then

(i) $E_{r+i s}\left|X_{r}\right| \geqq E_{i s}\left|X_{r}\right|$;

(ii) $E_{\imath s}\left|X_{r}\right| \leqq \Theta$.

Proof. (i) The distribution of $X_{\tau}$ under $P_{i s}$ can be found explicitly by Kakutani's theorem. It is given by a density $f_{i s}(t),-\infty<t<\infty$. Only the fact that $f$ is a symmetric function around 0 , which follows from the symmetry of a Brownian motion about a line through its point of origin, will be needed. The distribution of $X_{\tau}$ under $P_{r+i s}$ will be given by the 
density function $f_{r+i s}(t)=f_{i s}(t-r)$. Thus

$$
\begin{aligned}
E_{r+i s}\left|X_{\tau}\right| & =\int_{-\infty}^{\infty}|t| f_{r+i s}(t) d t \\
& =\int_{0}^{\infty}(|t-r|+|t+r|) f_{i s}(t) d t \\
& \geqq 2 \int_{0}^{\infty}|t| f_{i s}(t) d t=E_{i s}\left|X_{\tau}\right|,
\end{aligned}
$$

proving (i).

(ii) Let $\eta$ be the first time that $\left|Y_{t}\right|=s$. Then under $P_{0}, Z_{\eta}$ is distributed on the lines $\{z:|\operatorname{Im} z|=s\}$. Using (i), the strong Markov property, and the fact that the distribution of $X_{\tau}$ is the same under $P_{i s}$ and $P_{-i s}$, we have

$$
\begin{aligned}
E_{0}\left|X_{\tau}\right| & =E_{0} E_{Z_{\eta}}\left|X_{\tau}\right| \\
& \geqq E_{i s}\left|X_{\tau}\right| / 2+E_{-i s}\left|X_{\tau}\right| / 2 \\
& =E_{i s}\left|X_{\tau}\right|,
\end{aligned}
$$

proving (ii):

Lemma 2. Let $r$ be a real number and $\mu$ be a stopping time for $Z_{t}$. Then $E_{r}\left|X_{\min (\tau, \mu)}\right| \geqq \Theta\left(1-P_{r}(\mu<\tau)\right)$.

Proof. Clearly $E_{r+i s}\left|X_{\tau}-r\right|=E_{i s}\left|X_{\tau}\right|$. Thus, using Lemma 1 and the strong Markov property, we have

proving (ii).

$$
\begin{aligned}
\Theta & =E_{0}\left|X_{r}\right| \leqq E_{r}\left|X_{r}\right| \\
& \leqq E_{r}\left|X_{\min (\mu, \tau)}\right|+E_{r}\left|X_{\tau}-X_{\min (\mu, \tau)}\right| \\
& \doteq E_{r}\left|X_{\min (\mu, \tau)}\right|+E_{r} E_{Z_{\min (\mu, \tau)}}\left|X_{\tau}-X_{\min (\mu, \tau)}\right| \\
& =E_{r}\left|X_{\min (\mu, \tau)}\right|+E_{r} E_{i Y_{\min (\mu, \tau)}}\left|X_{\tau}\right| \\
& \leqq E_{r}\left|X_{\min (\mu, \tau)}\right|+\Theta P_{r}(\mu<\tau),
\end{aligned}
$$

3. Proof of Theorem 1. In this section $H_{t}, 0 \leqq t<\infty$, will stand for a complex Brownian motion started at 0 , and $d$ will be inf $\left\{t:\left|H_{t}\right|=1\right\}$. Define $F=U+i V$ on $D \cup A$ by $F(z)=\int_{A}\left[P\left(z, e^{i \theta}\right)+i Q\left(z, e^{i \theta}\right)\right] f\left(e^{i \theta}\right) d \theta$, $z \in D$, where $P$ and $Q$ are the Poisson and Poisson conjugate kernels, and $F(z)=f(z)+i \tilde{f}(z), z \in A . F$ is analytic in $D$ so, by a result of Levy, if $\gamma(t)=\int_{0}^{t}\left|F^{\prime}\left(H_{t}\right)\right|^{2} d t$ then $\gamma$ is almost surely strictly increasing and the process $\Gamma_{t}$ defined for $0 \leqq t \leqq \gamma(d)$ by $\Gamma_{\gamma(t)}=A_{\gamma(t)}+i B_{\gamma(t)}=F\left(H_{t}\right)$ is a standard Brownian motion started at $F(0)=\int_{A} f d m$ and stopped at the time $e=\gamma(d)$. [1] has a more complete discussion of this. Since $H_{d}$ is uniformly distributed on $A,\|f\|_{1}=E\left|A_{e}\right|$ and $m\{|\tilde{f}| \geqq 1\}=P\left(\left|B_{e}\right| \geqq 1\right)$. Let $\tau=\inf \{t:|B(t)|=1\}$, and let $v_{r}=\inf \left\{t:\left|H_{\imath}\right|=r\right\}$. Then $\left|A_{\min \left(t, \gamma\left(v_{r}\right)\right)}\right|, 0 \leqq t \leqq \infty$, is a continuous 
nonnegative bounded submartingale so that

$$
\begin{aligned}
\left.E \mid A_{\min \left(r_{\gamma},\left(v_{r}\right)\right.}\right) & \leqq E\left|A_{\gamma\left(v_{r}\right)}\right| \\
& =\int_{A}\left|\operatorname{Re} F\left(r e^{i \theta}\right)\right| d \theta / 2 \pi \leqq\|f\|_{1}=E\left|A_{e}\right| .
\end{aligned}
$$

Now $\lim _{r \rightarrow 1} v_{r}=d$, so $\lim _{r \rightarrow 1} \gamma\left(v_{r}\right)=e$, and thus

Thus, by Lemma 2 ,

$$
E\left|A_{\min (r, e)}\right| \leqq \liminf _{r \rightarrow 1} E\left|A_{\min \left(r, \gamma\left(v_{r}\right)\right)}\right| \leqq E\left|A_{e}\right| .
$$

$$
\begin{aligned}
m\{|\tilde{f}| \geqq 1\} & =P\left(\left|B_{e}\right| \geqq 1\right) \leqq P\left(\sup _{0 \leqq t \leqq e}\left|B_{t}\right|=1\right) \\
& =P\left(\left|B_{\min (\tau, e)}\right|=1\right)=1-P(e<\tau) \\
& \leqq \Theta^{-1} E\left|A_{\min (e, r)}\right| \leqq \Theta^{-1} E\left|A_{e}\right|=\Theta^{-1}\|f\|_{1} .
\end{aligned}
$$

For general $\lambda>0$,

$$
m(|\tilde{f}| \geqq \lambda)=m\left(\left|(f \mid \lambda)^{\sim}\right| \geqq 1\right) \leqq \Theta^{-1}\|f \mid \lambda\|_{1}=\Theta^{-1}\|f\|_{1} / \lambda,
$$

proving (i) and completing the proof of Theorem 1.

4. Proof of Theorem 2. The proof of Zygmund [5, Vol. 2, p. 256] that constants in some strong type inequalities for the Hilbert transform do not exceed the constants in the related conjugate function inequalities, can be readily modified to apply to the weak type $(1,1)$ inequality. Thus (2) holds. This can also be proved in a manner similar to the proof of (1).

Next, examples will be given to show that $\Theta^{-1}$ cannot be replaced by a smaller number in (2). Let $H(z)=(1+z)^{2} / 4 z$. Then $H(0)=\infty, H$ maps the half disc $D \cap U$ onto $U$ and maps the boundary of $D \cap U$ onto $R$. Let $K$ be the inverse of $H$. Then $K$ maps $[0,1]$ onto $\left\{e^{i \theta}, 0 \leqq \theta \leqq \pi\right\}$ and maps $R-[0,1]$ onto $(-1,1)$. Let $d$ represent the density of $K([0,1])$ on $A$ with respect to $m$, i.e., if $0<\alpha<\beta<2 \pi, \int_{\alpha}^{\beta} d\left(e^{i \theta}\right) d m\left(e^{i \theta}\right)=\mu\{r \in[0,1]$ : $\left.K(r) \in\left\{e^{i \theta}, \alpha<\theta<\beta\right\}\right\}$. We will not need the exact form of $d$ but only that it is a continuous function on $A$, which is easily checked. Let $d_{n}$ be the density of $K^{n}([0,1])$ on $A$, where the $n$ indicates product.

LEMMA 3. As $n$ approaches infinity, $d_{n}$ approaches 1 uniformly on $A$.

Proof. Let $0 \leqq \theta \leqq \varphi<2 \pi$.

We have

$$
\begin{aligned}
\left|d_{n}\left(e^{i \theta}\right)-d_{n}\left(e^{i \varphi}\right)\right| & =\mid n^{-1} \sum_{k=0}^{n-1} d\left(\exp \left[\frac{2 k \pi+\theta}{n}\right]\right) \\
& -n^{-1} \sum_{k=0}^{n-1} d\left(\exp \left[\frac{2 k \pi+\varphi}{n}\right]\right) \mid \\
& \leqq \sup _{0 \leqq k<n}\left|d\left(\exp \left[\frac{2 k \pi+\theta}{n}\right]\right)-d\left(\exp \left[\frac{2 k \pi+\varphi}{n}\right]\right)\right|,
\end{aligned}
$$


which approaches 0 as $n$ approaches infinity by the uniform continuity of $d$. Since $\int_{A} d_{n} d m=\mu[0,1]=1$, this establishes Lemma 3 .

Now let $G$ be as in the introduction. $G$ maps $D$ onto $S$, maps $A$ onto the boundary of $S$, maps $[-1,1]$ onto $[-i, i]$, and $G(0)=0$. Thus if we define $M_{n}$ by $M_{n}(z)=G\left(K^{n}(z)\right), M_{n}$ maps $U$ onto $S$, maps $[0,1]$ onto the boundary of $S$, and maps $R-[0,1]$ onto $(-i, i)$. Define $m_{n}(r)=$ $\operatorname{Re} M_{n}(r),-\infty<r<\infty$. Then since $M_{n}(r)=0$ if $r \notin[0,1]$, Lemma 3 implies

$$
\begin{aligned}
\left\|m_{n}\right\|_{1} & =\int_{[0,1]}\left|m_{n}(t)\right| d \mu(t)=\int_{A}\left|\operatorname{Re} G\left(e^{i \theta}\right)\right| d_{n}\left(e^{i \theta}\right) d m\left(e^{i \theta}\right) \\
& \rightarrow \int_{A}\left|\operatorname{Re} G\left(e^{i \theta}\right)\right| d m\left(e^{i \theta}\right)=\Theta .
\end{aligned}
$$

Since $M_{n}(z) \rightarrow 0$ as $|z| \rightarrow \infty$ it follows that $\tilde{m}_{n}(r)=\operatorname{Im} M_{n}(r),-\infty<r<\infty$, and thus $\mu\left\{\left|\tilde{m}_{n}\right| \geqq 1\right\}=\mu\{[0,1]\}=1$. Thus $\Theta^{-1}$ cannot be replaced by a smaller number in (2).

\section{REFERENCES}

1. B. Davis, On the distribution of conjugate functions of nonnegative measures, Duke Math. J. 40 (1973), 695-700.

2. J. L. Doob, Semimartingales and subharmonic functions, Trans. Amer. Math. Soc. 77 (1954), 86-121. MR 16, 269.

3. S. K. Pichorides, On the best values of the constants in the theorems of M. Riesz, Zygmund, and Kolmogorov, Studia Math. 46 (1972), 164-179.

4. E. M. Stein and G. Weiss, Introduction to Fourier analysis on Euclidean spaces, Princeton Math. Series, no. 32, Princeton Univ. Press, Princeton, N.J., 1971. MR 46 \#4102.

5. A. Zygmund, Trigonometric series. Vols. I, II, 2nd ed. reprinted with corrections and some additions, Cambridge Univ. Press, Cambridge, 1968. MR 38 \#4882.

Statistics Center, Rutgers University, New Brunswick, New Jersey 08903 